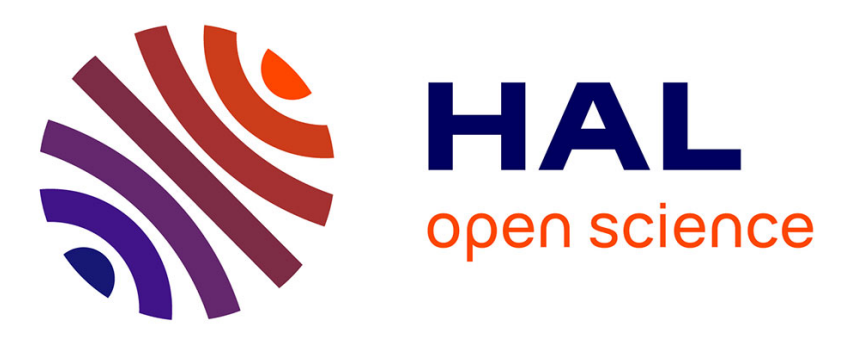

\title{
The use of photothermal techniques for thermal conductivity and thermal boundary resistance measurements of phase-change chalcogenides alloys
}

\author{
Jean-Luc Battaglia, Andrzej Kusiak, Kanka Ghosh
}

\section{- To cite this version:}

Jean-Luc Battaglia, Andrzej Kusiak, Kanka Ghosh. The use of photothermal techniques for thermal conductivity and thermal boundary resistance measurements of phase-change chalcogenides alloys. Journal of Applied Physics, 2021, 129 (5), pp.055106. 10.1063/5.0020983 . hal-03347651

\author{
HAL Id: hal-03347651 \\ https://hal.science/hal-03347651
}

Submitted on 17 Sep 2021

HAL is a multi-disciplinary open access archive for the deposit and dissemination of scientific research documents, whether they are published or not. The documents may come from teaching and research institutions in France or abroad, or from public or private research centers.
L'archive ouverte pluridisciplinaire HAL, est destinée au dépôt et à la diffusion de documents scientifiques de niveau recherche, publiés ou non, émanant des établissements d'enseignement et de recherche français ou étrangers, des laboratoires publics ou privés. 


\title{
The use of photothermal techniques for thermal conductivity and thermal boundary resistance measurements of phase-change chalcogenides alloys
}

\author{
Cite as: J. Appl. Phys. 129, 000000 (2021); doi: 10.1063/5.0020983 \\ Submitted: 6 July 2020 . Accepted: 13 January 2021 . \\ Published Online: $=2021$
}

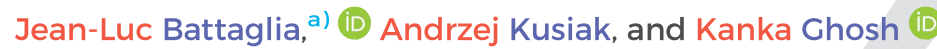

9 AFFILIATIONS

10 I2M Laboratory, UMR CNRS 5295, University of Bordeaux, 351 cours de la libération, 33400 Talence, France

11

Note: This paper is part of the Special Topic on Photothermics.

a) Author to whom correspondence should be addressed: jean-luc.battaglia@u-bordeaux.fr

\section{ABSTRACT}

16 This article presents three photothermal methods dedicated to the measurement of the thermal properties of chalcogenide alloys, used as a 7 central element in the new generations of non-volatile memory. These materials have two phases, amorphous and crystalline, possessing a sharp contrast in their electrical and thermal properties. In the crystalline phase, the properties also change very significantly with 9 temperature. The control of the temperature of the samples, the choice of transducers, and the time or frequency characteristic values of the photothermal excitation are thoroughly discussed. Each photothermal technique is described from the experimental point of view as well as from the inverse method, performed to identify the parameters of interest. The identified thermal properties mainly concern the thermal conductivity and the thermal resistance at the interfaces between the phase-change materials and the materials in contact as encountered in the production of the microelectronic memory device. Assessing various photothermal techniques, the study suggests that pulsed photothermal radiometry is the most effective method for sensitive high-temperature measurements of thermal properties of the phase-change materials.

Published under license by AIP Publishing. https://doi.org/10.1063/5.0020983

\section{I. INTRODUCTION}

The phase-change materials (PCMs) have been largely studied for several years because of their useful implementation within the field of non-volatile memories, ${ }^{1-4}$ leading to the phase-change RAM or PCRAM. Those chalcogenide binary or ternary alloys involve at least one chalcogenide element, generally $\mathrm{Te}$, and one or two other elements from columns 13, 14, and 15 as Ge, In, and Sb. The most well studied compounds are based on the In-Sb-Te and Ge-Sb-Te systems as reported in Fig. 1(a). These alloys are implemented in non-volatile memory devices because their electrical resistivity $R_{e}$ varies across several decades, according to the crystalline state of the alloys as showed in Fig. 1(b). In the amorphous state, the electrical resistivity is high and the material behaves as an 9 insulator, whereas in the crystalline state, the electrical resistivity is 0 very low and the material behaves like a metal. A bit, whether 0 or 411 or even intermediate, ${ }^{4,5}$ can be thus linked to this electrical state of the alloy. A continuous scaling of PCRAM devices is well observed 42 down-to the nanometer characteristic dimension ${ }^{6-8}$ across years. 43 Indeed, the technologies for the implementation of the alloy have not 44 ceased to evolve over time in order to reduce the transition times for 45 the phase change as well as the power consumption required for this 46 change. Thus, the first technologies have relied on the thin layer tech- 47 nology, which leads to a variation in the so-called "mushroom" pro- 48 gramming volume due to the shape of the half-sherry volume on the 49 heating electrode. ${ }^{9,10}$ More complex forms as micro-trenches have 50 also emerged. ${ }^{11}$ Finally, the latest developments aim to implement 5 the phase-change material in the form of nanowires ${ }^{12-14}$ whose diameter does not exceed a few nanometers or as PCM superlattices leading to the interfacial phase change memory technology. ${ }^{15}$

The thermal property measurement of PCM is a crucial step for their implementation in PCRAM. ${ }^{16-18}$ Indeed, knowing both the thermal properties, as a function of the temperature, and the crystalline state will allow the calculation of the electrical power 


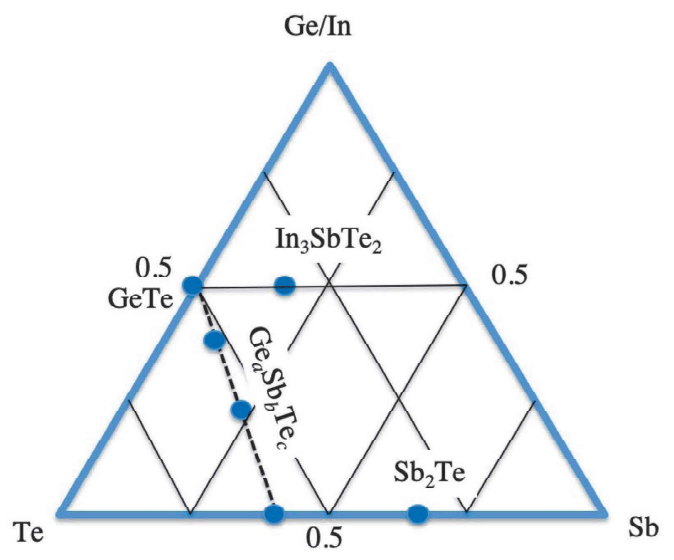

(b)

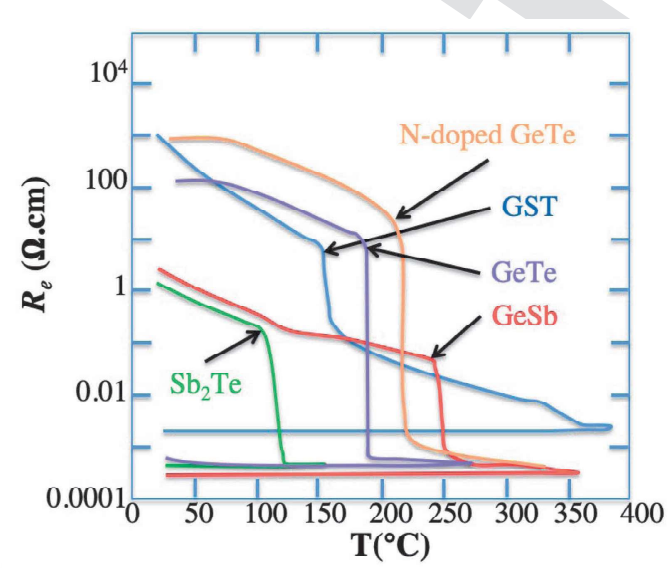

(b)

FIG. 1. (a) An overview of the phase-change alloys that have been investigated using the Ge-Sb-Te and In-Sb-Te ternary diagrams. (b) Resistivity as a function of temperature during a heating cycle for initially amorphous, as-deposited films of various phase-change materials (PCMs).

59 and associated transient waveform required for the phase change.

60 In addition, it also allows for the design of the memory cell in

61 order to avoid the thermal crosstalk effects with neighboring

62 cells. $^{19,20}$ The measurement of PCM thermal conductivity must be

63 performed over the entire temperature range including the

64 amorphous-crystalline phase transition and up to the melting tem-

65 perature. It is also well-established that the thermal boundary

66 resistance (TBR) at the interfaces between the PCM microvolume

67 and neighboring materials, such as the dielectrics and metal elec-

68 trodes, has a comparable influence than that of the thermal con-

69 ductivity on the heat transfer within the device. ${ }^{19,21-23}$ It must be

70 emphasized that, when the characteristic dimension of the system

71 becomes comparable to or less than the average mean free path of

2 the elementary heat carriers (phonons and electrons), the thermal

conductivity has no longer physical meaning from the point of

74 view of Fourier's law. In such a case, the measurement of the

thermal resistance or conductance of these nanostructured materi-

6 als is achievable. Typically, there are two major classes of methods

for the thermal characterization of materials deposited in thin

layers or nanostructured: contact methods and non-contact methods. Contact methods, as the $3 \omega^{24-26}$ and the scanning

thermal microscopy (SThM), ${ }^{27-34}$ have the advantage of having absolute measurements of flux and temperature. The major draw-

back of the contact methods is the presence of the additional unknown parameters, relating to the contact itself and the significant thermal inertia of the probes, which introduce difficulties in processing the very fast transients.

In this paper, we will discuss the implementation of PTR techniques as the periodic (MPTR) and pulsed (PPTR) photothermal radiometry within the infrared (IR) and the time domain thermore9 flectance (TDTR). All those PTR methods are based on the response to a thermal disturbance, generated as a heat flux $\varphi_{0}(t)$ at 91 the surface of the investigated material. This disturbance must be 2 small enough to fulfill the linearity requirement, regardless of the 93 value of the initial temperature $\left(T_{i}\right)$ of the material. The three methods are complementary since they involve different character- 94 istic time or frequency range by decades as well as different spatial 95 resolution. First, we present all the technological solutions provided 96 to carry out the temperature control of the sample. In particular, 97 we show the influence of the thermal loading of the sample on this 98 temperature control and on the choice of the most appropriate 99 optical-to-thermal transducer. Second, we present the most efficient 100 minimization techniques and more particularly show the contribu- 101 tion of inference techniques to predict the confidence domain of 102 the parameters identified with greater accuracy. It must be noted 103 that the inverse method is poorly discussed in the literature, 104 whereas it constitutes a fundamental step towards finding the 105 thermal properties, regarding mainly the identifiability of the 106 unknown parameters based on a sensitivity study. On the other 107 hand, the confidence domain of the identified parameters depends 108 not only on the statistical properties of the measured signal but 109 also on the minimization method used. Finally, the most advanced 110 experimental configurations for MPTR, PPTR, and TDTR are pre- 111 sented, focusing on the laser excitation time waveform and the 112 signal processing that involves both the model of the experiment 113 and the inverse procedure. The model is sometimes restricted to 114 the heat diffusion within the sample, whereas the complete acquisi- 115 tion chain affects the measured signal. A global model is thus 116 required that accounts with the all the experimental parameters. 117 The inverse method aims to minimize the difference between the 118 measured physical quantity and its value calculated from the model 119 discussed just before. The minimization is achieved by implement- 120 ing a set of suitable mathematical methods whose literature is 121 rich. ${ }^{35}$ On the other hand, it is clear that thermal conductivity and 122 TBR parameters are not always separately identifiable according to 123 the experimental configuration. Therefore, specific strategies have 124 to be implemented to separately distinguish TBR and thermal con- 125 ductivity, which can be done using the sensitivity analysis. In addi- 126 tion, it must also be said that some sample configurations, such as 127 thin films stacks or super lattices, also involve several interfaces, 128 
129 and it is extremely difficult to identify them separately. A typical

130 case for the PCRAM application is the stack formed by the metal

131 electrode, the PCM layer, and the dielectrics material that ensures

132 electrical and thermal insulation of the operating cell.

\section{II. GENERAL CONSIDERATIONS}

\section{A. Controlling the sample temperature}

The PCM layer is generally deposited on a $\mathrm{Si}$ wafer with $\mathrm{SiO}_{2}$ 36 thermal oxide at the surface. The adhesion of the PCM on $\mathrm{SiO}_{2}$ is 137 generally high and does not require an interfacial layer. Additional 138 layers, including the optical-to-thermal transducer, have to be con139 sidered, and finally, a stack of thin layers is obtained. The sample is

140 put inside a furnace that allows controlling the annealing tempera-

141 ture. The use of PTR methods requires the oven to be equipped

142 with an appropriate window that allows the passage of the pump

143 and probe laser for the methods based on thermoreflectance

144 (TDTR), as well as the laser and the IR radiation for the radiometry

145 methods (MPTR and PPTR). The designed furnace is represented

146 in Fig. 2(a). The window is $\mathrm{CaF}_{2}$ for MPTR and PPTR methods

147 since it is transparent for both the visible and infrared radiation as

148 showed in Fig. 2(a). For the TDTR method, the window is silica

149 glass since the pump and probe lasers work within the visible wave-

150 length as represented in Fig. 2(b). The silica glass transmittance 151 according to the radiation wavelength is reported in Fig. 2(b)

154 dient within the sample is low for low temperature, whereas it

155 increases drastically as the temperature increases. Indeed, convec-

156 tion and even more radiation are enhanced as the temperature gap

157 between the sample and the ambient is high. In addition, the

158 contact between the sample and the furnace is very weak, leading

159 to a high thermal resistance at the interface. For the MPTR and

160 PPTR configurations, there is no benefice from the $\mathrm{CaF}_{2}$ window to make greenhouse to occur since transmittance is high within IR. 161 It is then required performing a calibration of the sample surface 162 temperature for each type of transducer used (see Sec. II B) since 163 heat loss by radiation will depend on the emissivity of this material. 164 An illustration is given in Fig. 3(b) considering a sample capped 165 with a Pt layer. The emissivity of $\mathrm{Pt}$ is well measured ${ }^{36}$ and varies 166 according to wavelength and temperature as reported in Fig. 3(a). 167 Such dependence makes absolute temperature measurement at the 168 surface of the sample quite difficult. The second solution is to use 169 the glass transition temperature [see Fig. 1(b)] of the PCM as fixed 170 points on the experimental calibration curve. However, it must be 171 noted that the crystallization temperature could vary with the film 172 thickness when the former is low, typically of order of some nano- 173 meters. Therefore, the calibration with fixed points has to be per- 174 formed considering thick PCM layers, in general, more than 175 $100 \mathrm{~nm}$. Both the fixed point and the calibration from surface tem- 176 perature measurement are known, they are used simultaneously, 177 which allows one to achieve a better accuracy. This calibration is 178 not required anymore for the TDTR method since the silica glass 179 window makes the greenhouse to occur, and it is then observed 180 that the temperature at the sample surface is not significantly dif- 181 ferent from the set temperature of the furnace.

In order to limit the heat loss by convection and also the 183 sample oxidation, one can implement a secondary vacuum within 184 the furnace. However, given that it comes to lower the vaporization 185 temperature of the transducer material, a continuous deposition of 186 chemical species from the sample to the window of the oven is 187 observed. This contributes to modify the structure of the layers and 188 to obstruct the window at very short terms. A more efficient solu- 189 tion consists in producing a flow of argon gas within the oven 190 enclosure.

For all the characterizations performed using either the 192 MPTR, the PPTR, or the TDTR, the temperature ramp is 193 $25^{\circ} \mathrm{C} / \mathrm{min}$ and the stabilization time is $2 \mathrm{~min}$. The measurement 194
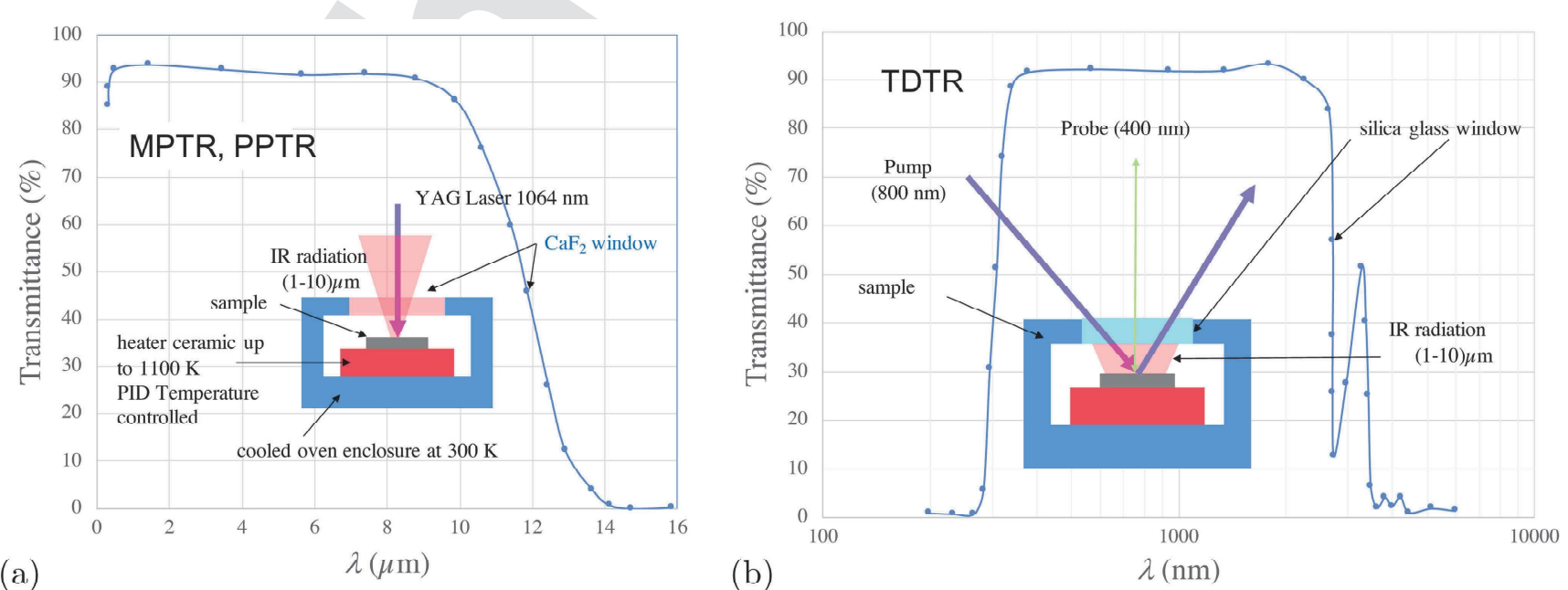

FIG. 2. (a) Designed furnace for the MPTR and PPTR methods at temperature up to $1100 \mathrm{~K} ; \mathrm{CaF}_{2}$ transmittance within the visible and IR wavelength range; (b) designed furnace for the TDTR method (transmittance of silica glass within the visible and IR wavelength range). 

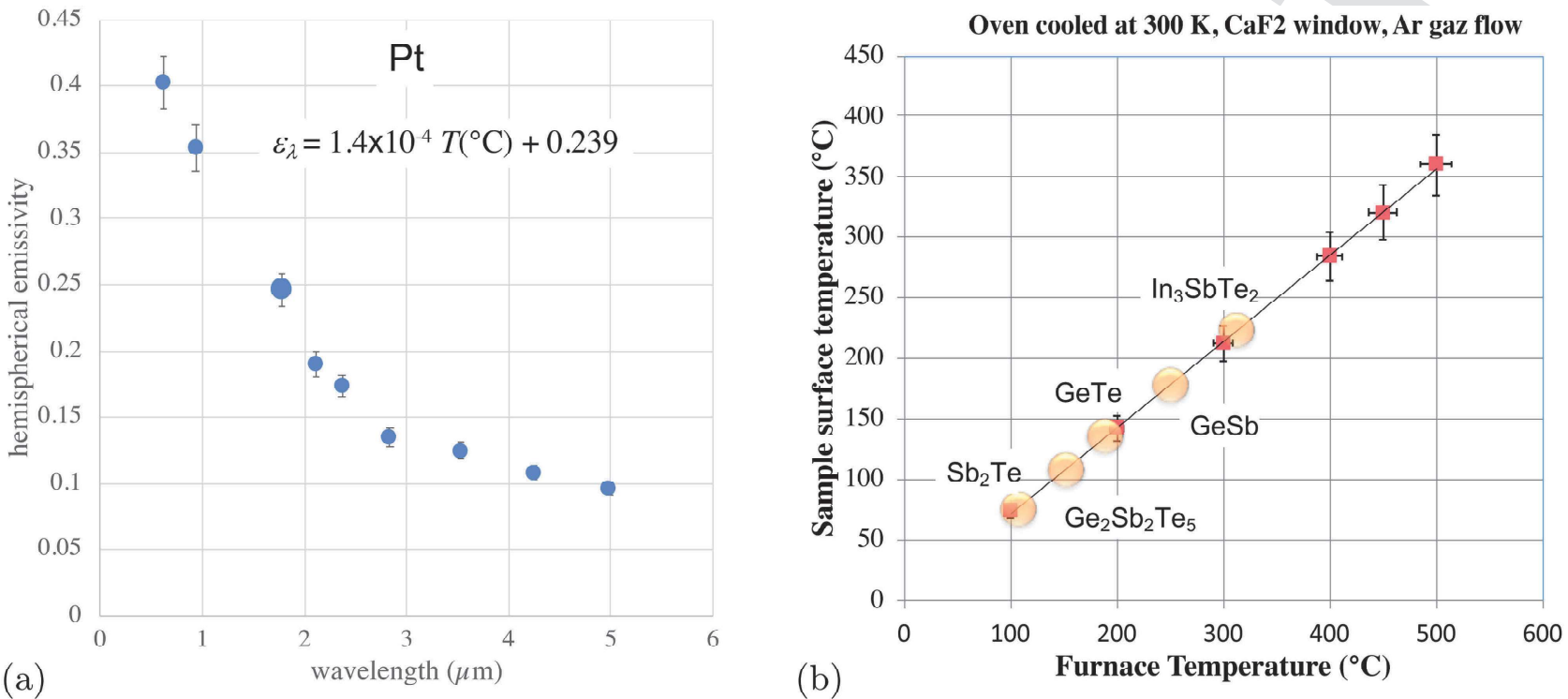

FIG. 3. (a) Pt spectral hemispherical emissivity according to wavelength and temperature; (b) surface temperature of the sample measured using an IR camera. The oven enclosure is cooled at $300 \mathrm{~K}$, there is a flow of Ar gas within the furnace and the window is $\mathrm{CaF}_{2}$. Crystallization temperature of different thick PCM alloys are reported on the plot.

195 time is about $10 \mathrm{~min}$ at each investigated temperature for the

196 MPTR and TDTR methods, whereas it is only about $20 \mathrm{~s}$ for the

197 PPTR one.

\section{B. Optical-to-thermal transducer}

The alloys constituting the PCMs are generally transparent to 200 the wavelength of the laser, regardless of the choice of the PTR 201 method. A full volume absorption does not make it possible to gen202 erate a temperature gradient within the layer to be characterized 203 and, therefore, to reach its thermal conductivity. On the other 204 hand, the PCM alloys being semiconductors, the carrier response is 205 very easily observed during the passage of the bandgap within the 206 IR signal. Thus, in order to control the absorption of the laser at 207 the surface of the material, it is common to deposit a layer, called 208 optical-to-thermal transducer whose role is to transform the inci209 dent photons into a surface heat flux. It must be said that the litera210 ture is often very discrete regarding the choice of the material 211 transducer for high-temperature characterization as well as its 212 thickness value. Most of the studies do not make a physicochemical 213 investigation of the layers as well as their interfaces after the appli214 cation of the high-temperature budget. The TiN material would be 215 very effective for high-temperature application, and it would be 216 also very interesting since it is generally used as the metal elec217 trodes within the PCRAM device. Unfortunately, this material is 218 not opaque within the visible wavelength. For MPTR and PPTR 219 radiometry techniques in the IR, it is strongly advised to search for 220 a transducer whose properties come closest to a blackbody. 221 Unfortunately, many candidate materials do not withstand high 222 temperatures. For instance, chromium is an excellent candidate for low temperatures given its high emission factor in IR. However, 223 when the temperature reaches $300^{\circ} \mathrm{C}$, cracks are observed on the 224 surface of the sample. After testing several coating layers, we found 225 that the only material that can withstand high-temperature levels, 226 without evaporating nor oxidizing, is platinum. However, as 227 showed in Fig. 3(a) the properties of platinum for both the absorp- 228 tion in the visible wavelength and the emission within the IR are 229 low. As reported in Fig. 4(a), Time of Flight-Secondary Ion Mass 230 Spectrometry (ToF-SIMS) has been performed at room temperature 231 (RT) for a $30 \mathrm{~nm}$ thick Pt layer deposited on a $210 \mathrm{~nm}$ amorphous 232 GeSbTe thick layer. The measurement was then done for the 233 annealed sample at $400^{\circ} \mathrm{C}$ when the PCM phase change has been 234 reached. It is thus observed a slightly diffusion of $\mathrm{Pt}$ within the 235 $\mathrm{Ge}_{2} \mathrm{Sb}_{2} \mathrm{Te}_{5}$ (GST) layer close to the interface. This observation 236 leads to limit the use of this technique to layers whose thickness is 237 large enough (more than $100 \mathrm{~nm}$ in practice) in order to not be sig- 238 nificantly affected by the transducer material diffusion at high tem- 239 perature. We also observed that the diffusion of species between 240 platinum and most chalcogen alloys (GeTe, SbTe, InSb, InSbTe) 241 remained very limited. It is obviously recommended to limit the 242 duration of the thermal budget of the investigated samples during 243 the experiment by carefully choosing the temperature ramp as well 244 as the duration of the measurement at each scanned temperature. 245

For thermoreflectance, we look for a material whose reflectiv- 246 ity as a function of the temperature is large. Pt is not suited for 247 such measurement and Au diffuses very quickly within the PCM 248 alloys as soon as the temperature increases. Al is generally the 249 material that presents satisfying properties in terms of temperature 250 dependent reflectivity and that can withstand thermal budget as 251 high as $400^{\circ} \mathrm{C}$ at the maximum without apparition of visible 252 


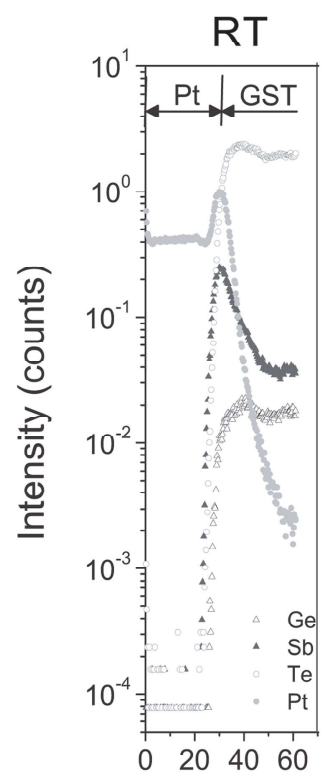

(a)
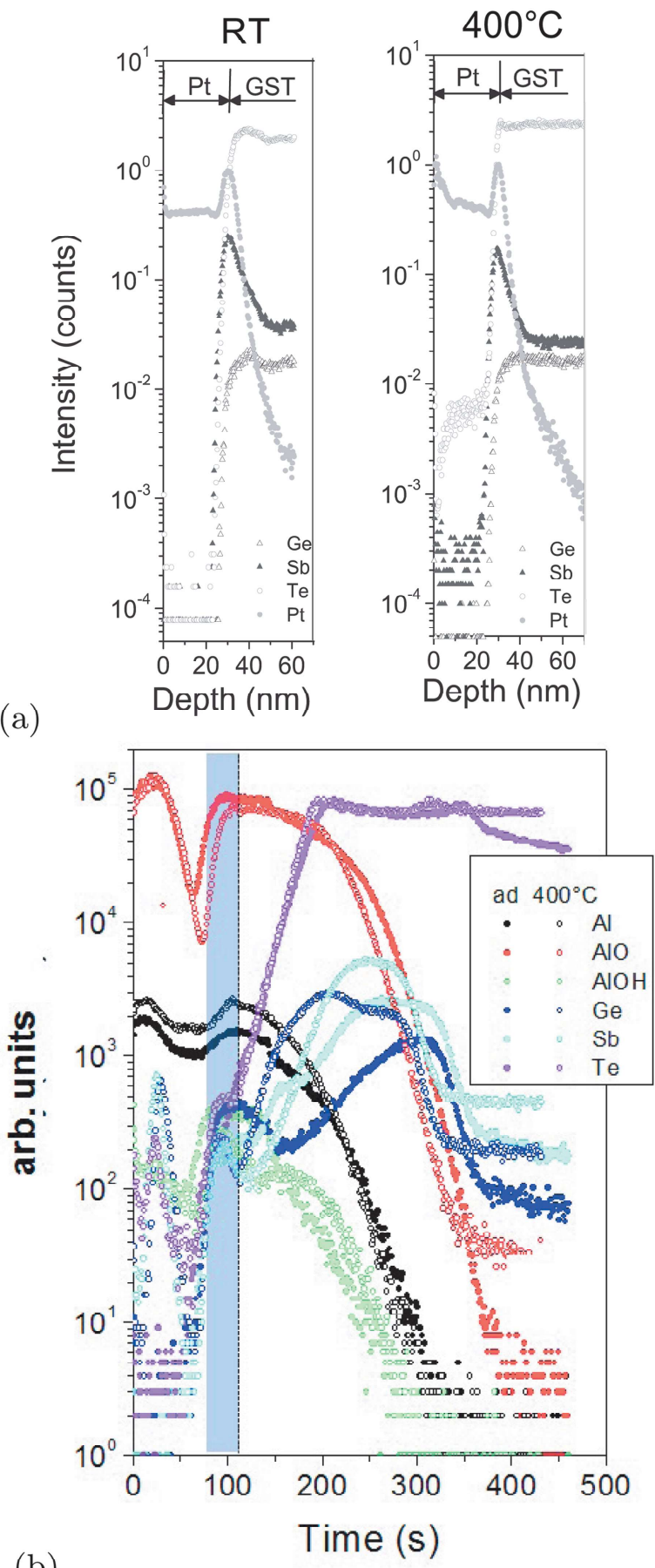

(b)

FIG. 4. (a) ToF-SIMS for the as-deposited amorphous GST with Pt capped layer and the annealed sample at $400{ }^{\circ} \mathrm{C}$ (GST in the hcp phase); ${ }^{37}$ (b) ToF-SIMS for the as-deposited (ad) amorphous GST with the Al capped layer and the annealed sample at $400^{\circ} \mathrm{C}$ (GST in the hcp phase). ${ }^{38}$ The dashed line locates the perfectly flat ideal interface, and the gray area evidenced the interface width. surface degradation. However, ToF-SIMS performed on amorphous 253 and $400{ }^{\circ} \mathrm{C}$ annealed sample evidences changes in depth profiles 254 for $\mathrm{Ge}, \mathrm{Sb}$, and $\mathrm{Te}$ species with diffusion into the Al layer and up 255 to the $\mathrm{Al}$ surface after annealing [Fig. 4(b)]. There is no Al diffu- 256 sion into GST, the apparent higher intensity seen in the annealed 257 sample being due to the concomitant $\mathrm{Ge}, \mathrm{Sb}, \mathrm{Te}$ diffusion at the 258 interface. Both information regarding surface roughness and mass 259 diffusion allow us defining the interface layer to be $0.9-3 \mathrm{~nm}$ thick. 260 Moreover, roughness uniformity allows us to conclude that the 261 mass amounts of GST and $\mathrm{Al}$ in the interface layer are close to 262 $\beta=60 \%$ and $(1-\beta)=40 \%$, respectively. This also leads us to 263 conclude that the method should be used for PCM layer whose 264 thickness is higher than $100 \mathrm{~nm}$.

A fundamental comment is about the fact that the thermal 266 budget applied to the sample for the characterization is far from 267 the way the PCM is heated during the PCRAM device operation. 268 This has not been clearly studied but the thermal load operation 269 will significantly change the way the interface is modified over 270 time. In the same vein, it is important to note that all the thermal 271 characterization experimental procedure reported in the literature 272 omit to specify the conditions of thermal load of the PCM materi- 273 als. Therefore, the thermal resistance measurement at the interfaces 274 between the PCM and neighbored layers reported within the litera- 275 ture has to be considered with high caution.

\section{Investigated depth within the sample}

277

When the heat flux is a periodic function of time with angular 278 frequency $\omega=2 \pi f$ (MPTR), the thermal diffusion length within 279 the expected material is a function of its thermal diffusivity $a$, that 280 is the ratio $k / \rho C_{p}$ of the thermal conductivity and the specific heat 281 per unit volume, and the frequency $f$ as: $z_{h}=\sqrt{a / \pi f}$. Similarly, 282 when the heat flux is generated as a pulse with duration $\tau$ (PPTR, 283 TDTR), the minimum investigated depth within the material is 284 $z_{h, \min }=\sqrt{a \tau}$. The typical heat penetration depth is illustrated in 285

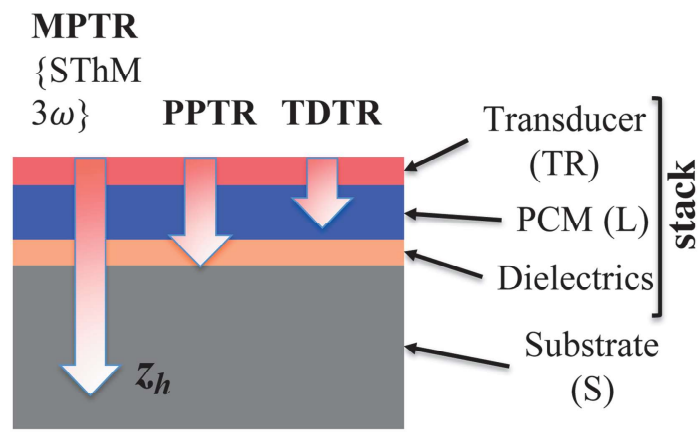

FIG. 5. The investigated heat penetration depth within the sample according to the technique used. The transducer thickness depends on the temperature range swept during the characterization, i.e., at ambient measurement the thickness transducer can be small enough $(10 \mathrm{~nm})$, whereas it must be larger at high temperature $(100 \mathrm{~nm})$ in order to resist to both thermomechanical constraints and evaporation. The $3 \omega$ and SThM techniques are also reported for information. Some additional interfacial layers can be considered with regard to adhesion purpose of the transducer. 
TABLE I. $R_{T}$ : thermal resistance involved within the heat transfer model considering the stack deposited on the substrate (S) and capped with the transducer (TR) as represented in Fig. 5 ; $t_{l}$ and $k_{l}$ are the thickness and the thermal conductivity of the PCM layer $(L) ; R_{K}$ is the intrinsic thermal resistances for the layers (TR and $\left.D\right)$ involved in the stacks with known thermal conductivity; TBR denotes the thermal resistance at each interface $i$ within the stack; $\Theta$ is the vector of identified parameters using the NLSQ method, $\mathbf{P}$ is the vector of identified parameters including uncertainties on known parameters using the Markov Chain Monte Carlo (MCMC) method (see the related section for the description of those experimental parameters)

\begin{tabular}{|c|c|c|c|}
\hline Method & $R_{T}$ & $\Theta$ & $\mathbf{P}$ \\
\hline MPTR & $\begin{array}{l}t_{l} / k_{l}+R_{K}+R_{c}, \text { with } R_{K}=\sum_{i} t_{i} / k_{i} \\
\quad \text { and } R_{c}=\sum_{i} T B R_{i}\end{array}$ & $R_{T}\left[k_{l}\right.$ and $R_{c}$ if $R_{T}=f\left(t_{l}\right)$ available $]$ & 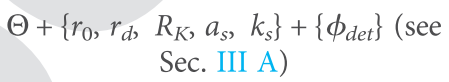 \\
\hline PPTR and TDTR & $R_{c}+t_{l} / k_{l}$, with $R_{c}=T B R(T R / L)$ & $k_{l}, R_{c}$ & $\begin{array}{c}\Theta+\left\{t_{l}\right\}+\left\{f_{m}, f_{c u t}, t_{d e l}\right\} \text { for the } \\
\text { PPTR (see III C) }\end{array}$ \\
\hline
\end{tabular}

286 Fig. 5, considering the three different methods. The thickness of 287 the layers constitutive of the stack being of the order of some tenth 288 of nanometers, only the thermal resistance of the investigated stack 289 deposited on the substrate can be reached by using the MPTR. This 290 thermal resistance includes both the intrinsic thermal resistance $291 t / k$ of the layers from the stack and the sum $R_{c}$ of the TBR at the 292 interfaces between the layers. A very important point in the use of 293 the experimental data for this technique is that here the substrate 294 defines the reference for these measurements since only the relative 295 variations of temperature can be measured. It is, therefore, impor296 tant to know the thermal properties of the substrate, over the entire 297 temperature range explored, with great accuracy. For the PPTR and 298 TDTR, it is expected that the thermal conductivity of the layer as 299 well as the TBR can be identified separately. Nevertheless, for these 300 two methods which lead to the measurement of the relative tem301 perature variation, it is the transducer that constitutes the reference 302 with respect to the use of the experimental data with regard to the 303 model. The properties of the transducers must, therefore, be known 304 with precision over the entire temperature range explored for these 305 two methods. Table I presents the parameters that can be identified 306 for the three methods. The three methods, therefore, appear to be 307 complementary because they lead to different information but 308 which, in fine, must overlap and lead, in particular, to the different 309 values of thermal conductivity of the PCM and of the thermal 310 resistances at the different interfaces.

\section{D. Identification procedure}

The identification of the seek parameters $\boldsymbol{\Theta}=\left[\alpha_{i}\right]$ ( $\alpha_{i}$ being 13 either a thermal resistance, a thermal conductivity, a TBR, or other 314 unknown parameters related to the experimental configuration used) is 315 based on several mathematical algorithms. The two most appropriate 316 classes of methods for this kind of inverse problem are the linear and 317 nonlinear least square (LSQ, NLSQ) techniques and the Bayesian 318 ones. $^{35}$ Of course, other techniques can be used (genetic algorithms, 319 particle swarm, etc.), but they will not provide additional information

320 than those obtained by the two classes of methods mentioned above.

321 Within the first class (as Newton-Gauss, Levenberg-Marquardt, ${ }^{39}$ or

322 trust-region-reflective algorithms ${ }^{40}$ ), the method will lead to minimize

323 the quadratic gap between the experimental data and those calculated

324 using a model of the heat transfer within the experimental configura-

325 tion. If the sensitivity functions $S_{Q}\left(\alpha_{i}\right)=\partial Q / \partial \alpha_{i}$ of parameters $\alpha_{i}$

326 relative to the measured quantity $Q$ (that is generally an absolute relative temperature or a phase-lag) are linearly independent, the mini- 327 mization of $J=\|\mathbf{Y}-\mathbf{Q}\|_{2}$, where $\mathbf{Q}=[\mathbf{Q}]_{\mathbf{N}}$ is the measurement 328 vector constituted from $N$ data, leads to a global minimum and then 329 to the optimal values for $\alpha_{i}$. On the other hand, this method allows 330 estimating the standard deviation of the identified values using the 331 covariance matrix for $\alpha_{i}$ at the end of the iterative minimization 332 process and the residuals $\mathbf{E}=\mathbf{Y}-\mathbf{Q}$ that are expected to be compara- 333 ble to the noise measurement assuming the model is unbiased. The 334 covariance matrix is $\operatorname{cov}(\boldsymbol{\Theta})=\left(\mathbf{S}^{T} \mathbf{S}\right)^{-\mathbf{P}}$, where vector $\mathbf{S}=\left[S_{Q}\left(\alpha_{i}\right)\right]_{N} .335$ It comes that the standard deviation of the identified parameters is 336 $\sigma\left(\alpha_{i} \mid Y\right)^{2} \sim \operatorname{cov}(\boldsymbol{\Theta}) \mathbf{E} / \sqrt{N}$. The main advantage of the non-linear least 337 square technique is the computation speed that is very fast when 338 approaching the minimum. The drawback of this approach is that it 339 assumes that other experimental parameters are known accurately, 340 which is not true in practice. Some uncertainties can be put on the 341 known parameters within the NLSQ technique assuming strong condi- 342 tions. Therefore, the Bayesian minimization technique can be effi- 343 ciently implemented assuming a standard deviation on the known 344 parameters. Indeed, in this method, all variables involved in the model, 345 formally gathered in column vector $\mathbf{P}(\boldsymbol{\Theta \subset} \mathbf{P})$, are considered random 346 variables. Information on variables is expressed as probability distribu- 347 tions. Each time a new information occurs for variables, it is combined 348 with the previously available information through the Bayes's theorem, 349 $\pi_{\text {posterior }}(\mathbf{P})=\pi(\mathbf{P} \mid \mathbf{Y})=\pi_{\text {prior }}(\mathbf{P}) \pi(\mathbf{Y} \mid \mathbf{P}) / \pi(\mathbf{Y})$, where $\pi_{\text {posterior }}(\mathbf{P})$ is 350 the posterior probability density, that is, the conditional probability of 351 the parameters $\mathbf{P}$ given the measurements $\mathbf{Y} ; \pi_{\text {prior }}(\mathbf{P})$ is the prior 352 density, that is, the coded information about the parameters prior to 353 the measurements; $\pi(\mathbf{Y} \mid \mathbf{P})$ is the likelihood function, which expresses 354 the likelihood of different measurement outcomes $\mathbf{Y}$ with $\mathbf{P}$ given; and 355 $\pi(\mathbf{Y})$ is the marginal probability density of the measurements, which 356 plays the role of a normalizing constant. This technique is generally 357 implemented as a Markov Chain Monte Carlo method, known as the 358 Metropolis-Hastings algorithm, ${ }^{41}$ so that inference on the posterior 359 probability becomes inference on the samples.

\section{EXPERIMENTAL TECHNIQUES}

\section{A. The MPTR technique}

The modulated photothermal radiometry method is a contact- 363 less measurement technique based on monitoring the emitted 364 infrared radiation from the surface of the sample consequently to a 365 periodic photothermal excitation $\varphi(t)$ provided by a laser. 


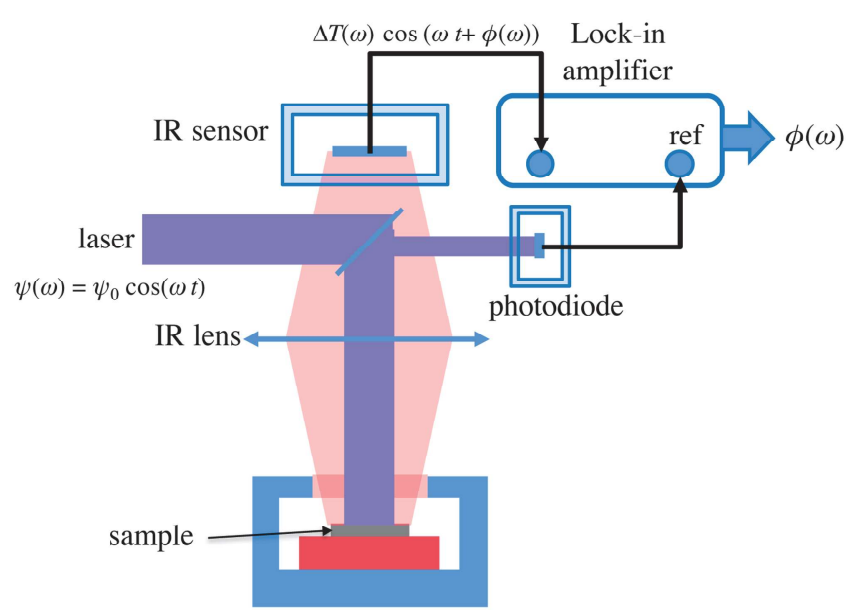

FIG. 6. The MPTR experimental setup.
Originally proposed in $1979,{ }^{42,43}$ the method has been exten368 sively improved for both the experimental ${ }^{44-46}$ and theoretical ${ }^{47-52}$ 369 aspects. The spatial distribution of the excitation can be uniform or 370 with more complex form (Gaussian, for example). The radiation 371 from the laser is absorbed by the surface of the sample, if opaque, 372 leading to a surface heat flux $\varphi$. This results in an increase $\triangle T$ at 373 the heating area leading to an infrared emitted radiation. 374 Considering a periodic heat flux $\varphi(t)=\varphi_{0} \cos (\omega t)$, the average 375 temperature increase over the measurement area at the surface of 376 the sample is constituted from a continuous and transient parts as: $377 \Delta T(t)=\triangle T_{0}+\Delta T_{\omega} \cos (\omega t+\phi)$. Assuming small temperature 378 increase, the transient part of the emitted radiation from the aimed 379 area can be linearized as: $\triangle M=4 \varepsilon \sigma_{s} \triangle T_{0}^{3} \triangle T_{\omega}$. The sketch of 380 the MPTR setup is presented in Fig. 6. A lock-in amplifier is used 381 to measure the signal at the IR detector and leads to the amplitude $382 A(\omega)$ and phase-lag $\phi(\omega)$ measurements. As said previously, for 383 thin layers of micrometer or sub-micrometer thickness deposited 384 on a substrate, the MPTR allows measuring, as for the $3 \omega$ method, 385 the global thermal resistance $R_{T}$ of the deposit. The calibration of 386 the amplitude vs the surface temperature requires knowing accu387 rately the surface emissivity $\mathcal{E}$ that comes to be a difficult task. 388 Since the phase $\phi(\omega)$ is very sensitive to the thermal resistance of 389 the layer, this measurement is used within the identification 390 process. The average temperature over the aimed area at the surface 391 of the sample is

$$
\overline{\triangle T}(\omega)=\varphi_{0}\left(Z_{\infty}(\omega)+R_{T}\right)
$$

392 with

$$
Z_{\infty}(\omega)=\frac{r_{0}^{2}}{k_{s} r_{d}} \sum_{n=0}^{M} \frac{J_{1}\left(\alpha_{n} r_{d}\right) e^{-\left(\frac{\alpha_{n} r_{0}}{2}\right)^{2}}}{\alpha_{n}^{2} \tanh \left(\sqrt{\alpha_{n}^{2}+\frac{j \omega}{a_{s}}} e_{t}\right) \sqrt{\alpha_{n}^{2}+\frac{j \omega}{a_{s}}} R^{2} J_{0}\left(\alpha_{n} R\right)^{2}}
$$

and $\alpha_{0}=0, \alpha_{n} R=\pi(n+1 / 4)-3 /(8 \pi(n+1 / 4))(n>0), r_{0}$ is 393 the laser beam radius, $r_{d}$ is the radius of the aimed area by the IR 394 detector, $k_{s}$ and $a_{s}$ are, respectively, the thermal conductivity and 395 thermal diffusivity of the substrate with thickness $e_{t}$ [for a semi- 396 infinite behavior $\tanh (\infty)=1]$. Finally, $J_{0}$ and $J_{1}$ are the kind 397 spessel functions of 0 and 1 order, respectively. The phase-lag 398 is then $\phi(\omega)=\arg \overline{\triangle T}(\omega)=\arctan (\operatorname{Im}(\overline{\triangle T}(\omega)) / \operatorname{Re}(\overline{\triangle T}(\omega))) .399$ The detector and associated amplifier involve a phase-lag $\phi_{\text {det }}$ that 400 increases linearly with the frequency $\omega$. The function $\phi_{\text {det }}(\omega)$ has to 401 be calibrated using a fast IR led and the model for the phase is, 402 therefore, $\widetilde{\phi}(\omega)=\phi(\omega)+\phi_{\text {det }}(\omega)$. Considering the measured value 403 $Y_{\phi}\left(\omega_{i}\right)$ of the phase-lag at different frequency $\omega_{i}(i=1, N)$, the 404 objective function is $J=\left\|\mathbf{Y}_{\boldsymbol{\phi}}-\boldsymbol{\Psi}\right\|_{2}$, where $\mathbf{Y}_{\boldsymbol{\phi}}=\mathbf{Y}_{\boldsymbol{\phi}}\left(\boldsymbol{\omega}_{\mathbf{i}}\right)$ and 405 $\boldsymbol{\psi}=\widetilde{\phi}\left(\omega_{i}\right)$ are respectively the measured and simulated phase at all 406 the investigated frequencies. The value of $R_{T}$ can be identified 407 using a nonlinear least square (NLSQ) technique as the Newton- 408 Gauss or Levenberg-Marquardt algorithm. In that case, the stand- 409 ard deviation on $R_{T}$ is achieved from the covariance matrix at the 410 end of the iterative process. In order to introduce uncertainties on 411 geometrical properties as $e_{t}, r_{0}$, and $r_{d}$ as well on the substrate 412 thermal properties $a_{s}$ and $k_{s}$ and the phase-lag $\phi_{\text {det }}$ of the detector 413 it is recommended to use the MCMC method with appropriate var- 414 iations. As described in Table I, the thermal resistance $R_{T}$ includes 415 the intrinsic thermal conductivity $k_{l}$ of the PCM layer $(L)$ as well as 416 the thermal resistance of other layers (TR and $D$ ) constituting the 417 stack presented in Fig. 5, and finally, the sum $R_{c}$ of the thermal 418 resistances at the interfaces between layers of the stack. The MPTR 419 allow the determination of $R_{T}$ and that of $k_{l}$ and $R_{c}$ if the experi- 420 ment can be repeated with different values of the thickness $t_{l}$. In 421 such a case, a linear regression is applied to the resistance measure- 422 ments as a function of the thickness for each temperature of the 423 PCM that leads to the value of the two parameters $\left(1 / k_{l}\right.$ being the 424 slope and $R_{c}$ the value at the origin). This approach has the advan- 425 tage of increasing the accuracy of the measurement on the two 426 parameters by confirming a linearity relationship. The major draw- 427 back remains the obligation to fabricate additional samples and the 428 duration of the characterization experiments also becomes much 429 longer.

The method has been used to measure the temperature depen- 431 dent thermal conductivity of several chalcogenide alloys: $\mathrm{Ge}_{2} \mathrm{Sb}_{2} \mathrm{Te}_{5} 432$ (GST), ${ }^{37} \mathrm{GeTe},{ }^{53} \mathrm{C}$-doped GeTe, ${ }^{54}$ and $\mathrm{In}_{3} \mathrm{Sb}_{1} \mathrm{Te}_{2} .{ }^{55}$ It has been 433 also used to investigate the TBR at the $\mathrm{SiO}_{2}-\mathrm{GST}$ interface ${ }^{37}$ and 434 the role of $\mathrm{Ti}$ at the interface between TiN (the metal electrode in 435 the PCRAM) and GST. ${ }^{56}$

\section{B. The TDTR technique}

Originally designed to study ultrafast phenomena, the time 438 domain thermoreflectance (TDTR) has been implemented within 439 the framework of thermal characterization. ${ }^{57}$ The technique has 440 been largely improved up to nowadays. ${ }^{58-60}$ A high-energy picosec- 441 onds or even femtoseconds laser produces a very short pulse with 442 high frequency repetition rate $f_{m}$. The beam is split as a low-energy 443 probe beam and a high-energy pump beam. Pump and probe 444 beams have generally the same diameter and are superimposed at 445 the sample surface. Drawbacks in using a mechanical stage as the 446 optical delay line can be avoided by using the heterodyne 447 
448 method. ${ }^{61}$ The pump is modulated at a low frequency that allows 449 the accurate extraction of the measured periodic change of surface 450 reflectivity using a lock-in amplifier that measure the voltage drop 451 at the photodiode. The probe is continuously delayed from the 452 pump with time $\tau$ in the nanoseconds time range. The pump is 453 thus used to heat the sample surface, whereas the probe is used to 454 monitor the change of surface reflectivity $\Delta R / R_{0}$ using a photodi455 ode. Assuming this change is proportional to that of the tempera-

456 ture, it is then obtained the quantity of interest for the Q4 457 identification process (Fig. 7).

458 An optical-to-thermal transducer is used that is generally gold 459 or aluminum. The diameter of the pump is larger than the PCM 460 film thickness leading to consider one-dimensional heat diffusion 461 within the sample. On the other hand, given to the very fast transi462 ent excitation and observation time, the TDTR method leads to 463 exploring only the PCM layer and the interface with the transducer 464 layer. The model that allows to simulate the measured signal by the 465 lock-in has to account also with the modulation of the laser beam, 466 and it is finally obtained

$$
D(t)=\sum_{n=-\infty}^{+\infty} \overline{\triangle T}\left(2 \pi\left(\frac{n}{\tau}+f_{m}\right)\right) \exp \left(-\frac{j 2 \pi n t}{f_{m}}\right)
$$
469 sider the layer at a uniform temperature at each time, one has

$$
\overline{\triangle T}(\omega)=\varphi_{0}\left(1 / E_{l} \sqrt{j \omega}+R_{T}\right),
$$

470 where $E_{l}=\sqrt{k_{l} \rho_{l} C_{p, l}}$ is the effusivity of the PCM layer. Since the 471 heat flux absorbed by the surface from the pump is not known in 472 practice and that it is only measured a relative variation of the tem473 perature at the surface, a normalized function $\widetilde{D}(t)$ of $D(t)$ with 474 respect to its value at $t$ chosen between 0 and $\tau$ is considered. Both

$475 R_{T}$ and $k_{l}$ can be identified using either the NLSQ or the MCMC

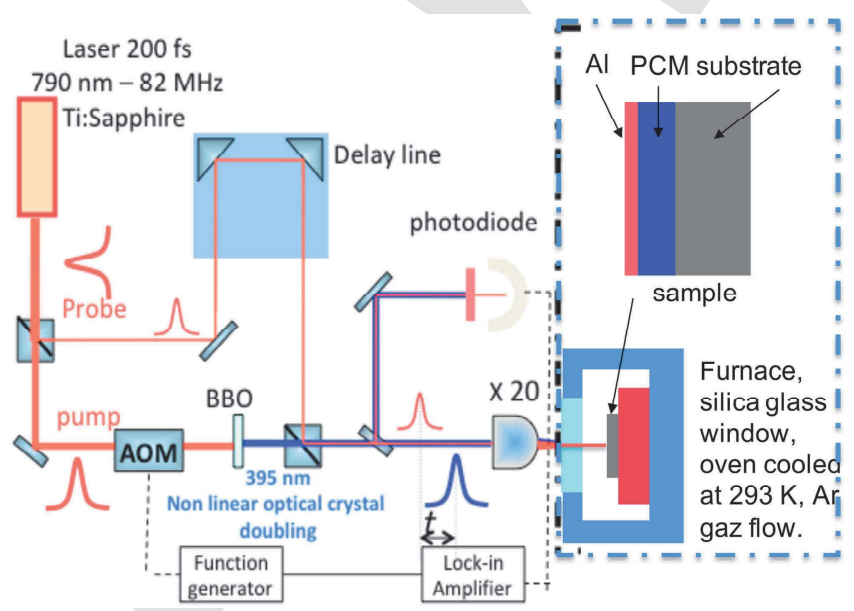

FIG. 7. Sketch of TDTR experimental setup. technique, although the former is recommended in order to intro- 476 duce an uncertainty on the modulation frequency $f_{m}$.

The method has been used to measure the temperature depen- 478 dent thermal conductivity of several PCMs, GST, ${ }^{62-64} \mathrm{Sb}_{2} \mathrm{Te}_{3},{ }^{65}{ }_{479}$ and GeTe. ${ }^{66}$ It has been also used to measure the TBR at the 480 GST-Al interface, ${ }^{38}$ the TBR at the TiN-GST interface ${ }^{21,23,67}$ and 481 the influence of fullerene $\mathrm{C}_{60}$ at the GST-TiN interface. ${ }^{68}$

Unfortunately, the material used as the transducer ( $\mathrm{Al}$ or $\mathrm{Au}$ ) 483 are not suited to work at high temperature, the maximum admissi- 484 ble temperature being of the order of $300^{\circ} \mathrm{C}$, beyond which crack- 485 ing as well as a strong atomic diffusion is observed. Given that we 486 are seeking to develop PCMs with a high crystallization tempera- 487 ture for high-temperature applications, the TDTR method turns 488 out to be less and less suitable for this type of characterization.

\section{The front face PPTR technique}

The approach is similar to that of the MPTR but in that case 491 the photothermal source is continuously emitting nanoseconds 492 heat pulses at frequency $f_{m}$ ranging from $1 \mathrm{kHz}$ up to $100 \mathrm{kHz} .493$ Once the steady periodic regime is reached, the signal measured by 494 the IR detector, which is proportional to the front face temperature, 495 is recorded after every pulses and averaged with the previous 496 average signal. In comparison with the classical flash technique, ${ }^{69} 497$ and even accounting with all the successive improvements, ${ }^{70-78} 498$ averaging the recorded signal leads to a significant improvement of 499 the signal noise ratio $^{79}$ since the standard deviation of measured 500 values of noise is reduced by $\sqrt{N_{s}}$, where $N_{s}$ denotes the number of 501 pulses used to perform the average. A fast IR detector $(20 \mathrm{MHz}$ and 502 nanoseconds rise time) is implemented. The optical arrangement is 503 quite similar to that of the MPTR in order to make the image of 504 the sensitive element of the detector on the heated area by the 505 laser. The sketch of the experimental setup is represented in Fig. 8.506

Accounting with the periodic repetition, it is obtained that the 507 signal measured between two successive pulses, when the stationary 508 regime is reached, is expressed from the average temperature on the 509

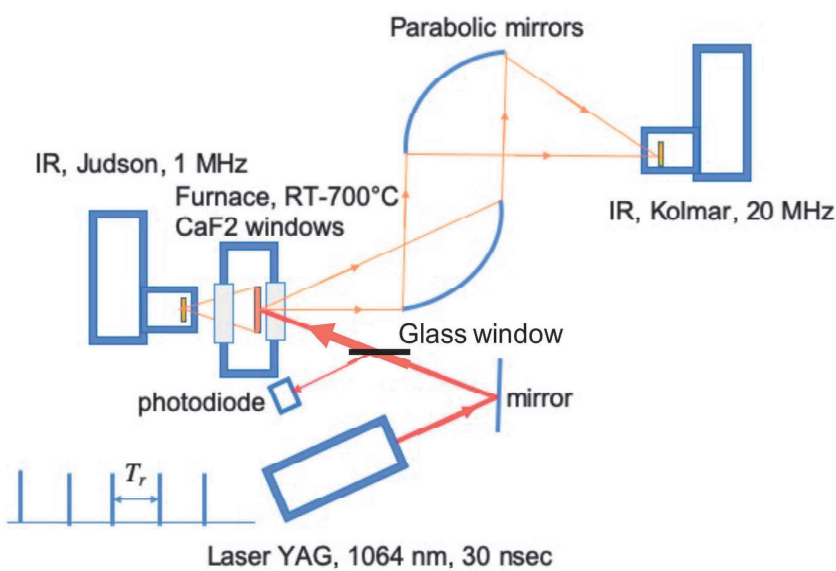

FIG. 8. Sketch of the PPTR experimental setup. 
510 aimed area as

$$
D(t)=\sum_{n=-\infty}^{+\infty} \overline{\triangle T}\left(2 \pi f_{m}\right) \exp \left(-\frac{j 2 \pi n t}{f_{m}}\right)
$$

512 If the layer behaves as a semi-infinite medium for the value of $513 f_{m}$, meaning $e_{l} \gg \sqrt{a_{l} / \pi f_{m}}$, then $\overline{\triangle T}(\omega)$ is given by relation (1) 514 with $\tanh (\infty)=1$ and replacing $k_{s}$ and $a_{s}$ by the properties of the 515 layer, i.e., $k_{l}$ and $a_{l}$. The thermal resistance $R_{T}=e_{T R} / k_{T R}+R_{c}$ is 516 the sum of the intrinsic resistance of the transducer layer with the 517 contact resistance $R_{c}$ at the interface between the transducer and 518 the PCM layer. Since the laser beam radius is much large than the 519 layer thickness, the heat transfer is one dimensional and the rela520 tion (1) can be simplified to obtain the same expression of $\overline{\triangle T}(\omega)$ 521 than that of the TDTR technique, e.g., relation (4). Hence, $E$ and $522 R_{T}$ can be identified using the minimization algorithms discussed 523 below. In case, the heat penetration depth is larger than the PCM 524 layer thickness but still less that the laser beam radius, a model 525 based on the thermal impedance network $\operatorname{method}^{80}$ can be 526 implemented. 28 represented in Fig. 9 with $Z_{1}(\omega)=(\cosh (\beta e)-1) /\left(k_{l} \beta \sinh (\beta e)\right)$, $529 Z_{3}(\omega)=1 /\left(k_{l} \beta \sinh (\beta e)\right), \quad \beta=\sqrt{j \omega / a_{l}}, \quad E_{s}=\sqrt{k_{s} \rho_{s} C_{p, s}}$, and

$530 Z_{s}(\omega)=1 / E_{s} \sqrt{j \omega}$. Therefore, the temperature at the aimed area is

$$
\overline{\triangle T}(\omega)=\varphi_{0}\left(\frac{1}{\frac{1}{Z_{3}(\omega)}+\frac{1}{Z_{1}(\omega)+Z_{s}(\omega)}}+Z_{1}(\omega)+R_{T}\right) .
$$

532 Since the heat flux absorbed by the surface from the laser is 533 not known in practice and that it is only measured a relative varia534 tion of the temperature at the surface, a normalized function $\widetilde{D}(t)$ 535 of $D(t)$ with respect to its value at $t$ chosen between 0 and $1 / f_{m}$ is 536 considered. The model has to account with the frequency trans537 forms $H_{\varphi}(\omega)$ of the pulse transient waveform and the transfer func538 tion $H_{\operatorname{det}}(\omega)$ of the detector that is considered as a delayed 539 first-order low-pass filter with cut-off frequency $f_{\text {cut }}$ and delay $t_{d e l}$.

540 It leads to replace $\overline{\triangle T}(\omega)$ by the double convolution product $541 \overline{\triangle T}(\omega)^{\star} H_{\varphi}(\omega)^{\star} H_{\text {det }}(\omega)$.

542 Both $R_{T}$ and $k_{l}$ can be identified using the NLSQ algorithm, 543 although it is rather recommended to use the Bayesian technique, 544 which allows introducing uncertainties on $\left\{t_{l}, f_{m}, f_{c u t}, t_{d e l}\right\}$. The

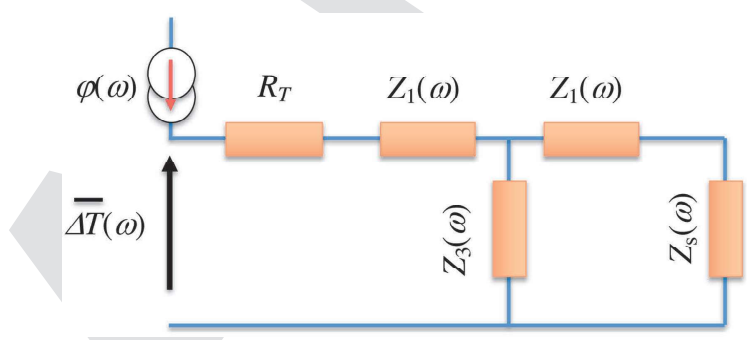

FIG. 9. Heat transfer model within the front face PPTR configuration considering the heat penetration depth is larger than the PCM thickness.
PPTR method has advantages for the characterization of high- 545 temperature PCM layers. The first is that the sensitivity of the 546 method remains two orders of magnitude above that based on 547 thermo-reflectivity. The second is the possibility of using trans- 548 ducers whose chemical affinity with PCMs is low (as Pt) and which 549 resist well at high temperatures, at least up to $500^{\circ} \mathrm{C}$. Finally, the 550 method, in its current state, makes it possible to explore layers of a 551 few tenths to hundred nanometers without the contact with the 552 lower layers being considered. Considerable technological progress 553 now makes it possible to use detectors whose acquisition frequency 554 can reach $100 \mathrm{MHz}$. This is still insufficient to compete with the 555 TDTR method, but it will make it possible to characterize layers of 556 PCM of a few tens of nanometers without having to diffuse into 557 the lower layers. It, therefore, seems that real progress can be 558 obtained by using the PPTR method for the characterization of 559 thin layers of PCM as a function of temperature in the future. The 560 method is quite recent and has been only used to measure the 561 thermal conductivity of the amorphous GeTe alloy and the TBR at 562 the interface with $\mathrm{Pt}^{81}$

\section{ILLUSTRATIONS}

564

We give in Fig. 10 the results obtained by using the three 565 methods described previously for three phase-change alloys, 566 namely, $\mathrm{Ge}_{2} \mathrm{Sb}_{2} \mathrm{Te}_{5}$ (GST), $\mathrm{In}_{3} \mathrm{Sb}_{1} \mathrm{Te}_{2}$ (IST), and GeTe. The PPTR 567 method was used for GeTe, the MPTR method was used for the 568 IST and the TDTR method was used for the GST. For each alloy, 569 we start from the amorphous state and we perform a measurement 570 for each prescribed temperature of the sample. The standard devia- 571 tion for each identified value is also reported in the figure, and it 572 must be also accounted with the $5 \%$ of uncertainty for the annealed 573 temperature of the sample (only represented for GeTe in the figure) 574 As one might expect, the thermal conductivity of materials in the 575 amorphous state does not change with temperature. Then, we 576 observe for the three systems a glass transition to the crystalline 577

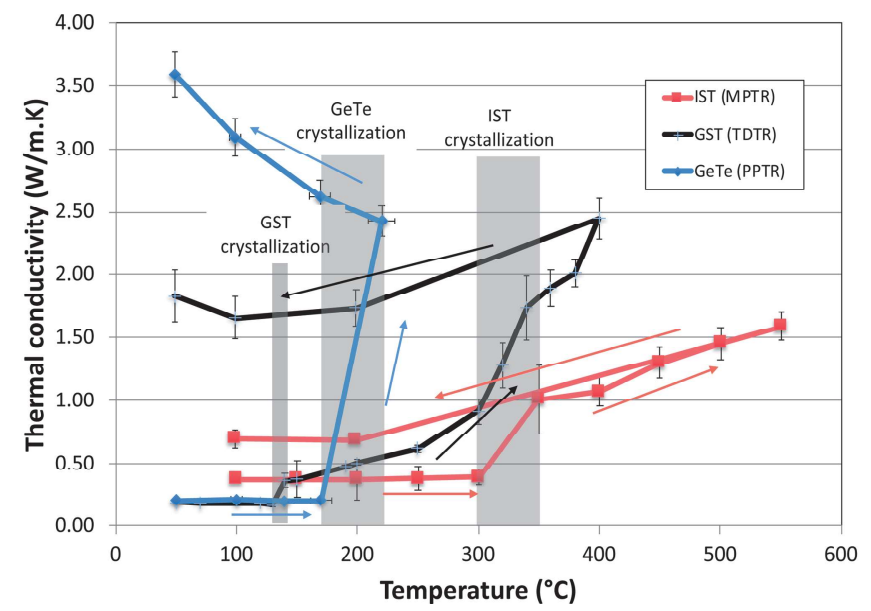

FIG. 10. Illustration of the use of the three techniques for the thermal conductivity measurement of $\mathrm{Ge}_{2} \mathrm{Sb}_{2} \mathrm{Te}_{5}$ (GST), $\mathrm{In}_{3} \mathrm{Sb}_{1} \mathrm{Te}_{2}$ (IST), and GeTe. 
578 state as well as a variation of the conductivity of the crystalline 579 phase with the temperature, the slope of which being essentially 580 linked to the electronic behavior. These variations are in agreement 581 with the electrical resistivity measurements as we presented them 582 in Fig. 1(b) in the Introduction section. A mainly striking result is 583 above all the uncertainty on the glass transition temperature $T_{c}$ 584 value, which is within the shaded areas in the figure. In fact, the 585 amorphous phase being very unstable, the transition to the crystal586 line state may appear during a measurement at a temperature 587 slightly below $T_{c}$, whatever the technique used. For information the 588 exact phase change temperature are $150^{\circ} \mathrm{C}$ for GST, $180^{\circ} \mathrm{C}$ for $589 \mathrm{GeTe}$, and $320^{\circ} \mathrm{C}$ for IST. It is, therefore, essential to carry out the 590 measurement, at a given temperature, in the shortest possible time 591 in order to minimize the transition from the amorphous state to 592 the crystalline state when one approaches the transition. It should 593 also be noted that the shorter the thermal loading time, the more 594 the risks of degradation of the transducers, as well as the diffusion 595 of species between layers are minimized. The PPTR method is the 596 most efficient for this purpose since only one transient measure597 ment is required at a given temperature, while the MPTR and 598 TDTR methods require several.

\section{V. CONCLUSION}

600 601 achieved for three photothermal radiometry methods used for the 602 measurement of thermal properties as the thermal conductivity of 603 phase-change chalcogenide alloys and related thermal boundary 604 resistances. Those methods are complementary not only in terms 605 of improving the accuracy of the seek parameters but also to dis606 criminate easily the thermal resistance at the interfaces between the 607 PCM and the adjacent layers that are the metallic dielectrics and 608 electrodes of the PCRAM cell. As we have shown, these methods 609 are much more effective than contact methods ( $3 \omega$ and SThM) 610 when we want to measure the changes in these thermal properties 611 at high temperatures, above the phase-change temperature. We 612 have particularly emphasized in this paper on the aspects linked to 613 the implementation of devices for controlling the temperature of 614 the sample, the choice of optical-thermal transducers, and the evo615 lution of materials as a function of temperature. A conclusion to 616 this part is that the thermal budget undergone by the samples will 617 have a significant role on the evolution of the sample and in fact 618 on the thermal properties. For fairly thick layers of the PCM, this 619 especially has repercussions on the value of the TBR, given the 620 strong interface variables which are observed in terms of composi-

621 tion and equivalent thickness. This also suggests that a thermal 622 characterization of thin layers of PCM at high temperature should 623 systematically be preceded and followed by a physicochemical char624 acterization (ToF-SIMS, Raman, DRX), which takes account of the 625 possible modifications undergone by the sample. This is still too 626 rarely done systematically in most published studies on PCM. We 627 have also shown the utility of using techniques for identifying 628 unknown parameters which take into account the uncertainty on 629 all the other known parameters, whether they are related to the 630 experimental method or to the properties of materials, other than 631 the PCM, constituting the sample. In this, the MCMC method is in 632 our opinion the most efficient. Finally, we have shown that the
PPTR technique becomes a credible alternative to the TDTR 633 method for high temperatures where transducer materials have to 634 withstand intense thermal loads. Further improvement is needed in 635 this area so that the observation times allow an investigation of the 636 PCM layer alone. However, recent technological developments to 637 make IR detectors capable of operating at frequencies above 638 $100 \mathrm{MHz}$ can further open up avenues of real applications.

\section{AUTHORS' CONTRIBUTIONS}

All authors contributed equally to this work.

\section{ACKNOWLEDGMENTS}

This project has received funding from the European Union's 643 Horizon 2020 research and innovation program under Grant 644 Agreement No. 824957 ("BeforeHand:" Boosting Performance of 645 Phase Change Devices by Hetero- and Nanostructure Material 646 Design).

\section{DATA AVAILABILITY}

The data that support the findings of this study are available 649 within the article.

\section{REFERENCES}

1S. R. Ovshinsky, Phys. Rev. Lett. 21, 1450 (1968).

${ }^{2}$ N. Yamada, E. Ohno, K. Nishiuchi, N. Akahira, and M. Takao, J. Appl. Phys. 653 69, 2849 (1991).

${ }^{3}$ D. Lencer, M. Salinga, B. Grabowski, T. Hickel, J. Neugebauer, and M. Wuttig, 655 Nat. Mater. 7, 972 (2008)

${ }^{4}$ S. Raoux, F. Xiong, M. Wuttig, and E. Pop, MRS Bull. 39, 703 (2014). ${ }^{\mathbf{5}}$ W. Zhang, R. Mazzarello, M. Wuttig, and E. Ma, Nat. Rev. Mater. 4, 150658 (2019).

${ }^{6}$ A. Pirovano, A. L. Lacaita, A. Benvenuti, F. Pellizzer, S. Hudgens, and R. Bez, in 660 IEEE International Electron Devices Meeting 2003 (IEEE, 2003), pp. 29.6.1- 661 29.6.4.

${ }^{7}$ S. Raoux, G. W. Burr, M. J. Breitwisch, C. T. Rettner, Y. Chen, R. M. Shelby, 663 M. Salinga, D. Krebs, S. Chen, H. Lung, and C. H. Lam, IBM J. Res. Dev. 52, 465664 (2008).

${ }^{8}$ R. Annunziata, P. Zuliani, M. Borghi, G. De Sandre, L. Scotti, C. Prelini, 666 M. Tosi, I. Tortorelli, and F. Pellizzer, in 2009 IEEE International Electron 667 Devices Meeting (IEDM) (IEEE, 2009), pp. 1-4.

9. H. Yi, Y. H. Ha, J. H. Park, B. J. Kuh, H. Horii, Y. T. Kim, S. O. Park 669 Y. N. Hwang, S. H. Lee, S. J. Ahn, S. Y. Lee, J. S. Hong, K. H. Lee, N. I. Lee, 670 H. K. Kang, U.-I. Chung, and J. T. Moon, in IEEE International Electron Devices 671 Meeting 2003 (IEEE, 2003), pp. 37.3.1-37.3.4.

${ }^{10}$ S. Tyson, G. Wicker, T. Lowrey, S. Hudgens, and K. Hunt, in 2000 IEEE 673 Aerospace Conference. Proceedings (Cat. No.00TH8484) (IEEE, 2000), Vol. 5, 674 pp. 385-390.

11 F. Pellizzer, A. Pirovanc, F. Ottogalli, M. Magistretti, M. Scaravaggi, P. Zuliani, 676 M. Tosi, A. Benvenuti, P. Besana, S. Cadeo, T. Marangon, R. Morandi, R. Piva, 677 A. Spandre, R. Zonca, A. Modelli, E. Varesi, T. Lowrey, A. Lacaita, 678 G. Casagrande, P. Cappelletti, and R. Bez, in Digest of Technical Papers. 2004679 Symposium on VLSI Technology, 2004 (IEEE, 2004), pp. 18-19. 680

${ }^{12}$ M. Longo, R. Fallica, C. Wiemer, O. Salicio, M. Fanciulli, E. Rotunno, and 681 L. Lazzarini, Nano Lett. 12, 1509 (2012). 682

${ }^{13}$ B. Yu, X. Sun, S. Ju, D. B. Janes, and M. Meyyappan, IEEE Trans. 683 Nanotechnol. 7, 496 (2008). ${ }^{\mathbf{1 4}}$ F. Xiong, M.-H. Bae, Y. Dai, A. Liao, A. Behnam, E. Carrion, S. Hong, 685 D. Ielmini, and E. Pop, Nano Lett. 13, 464 (2013).

\section{.} . .

\section{4} , .

\section{.} .

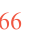
668 . 
$687{ }^{15}$ R. E. Simpson, P. Fons, A. V. Kolobov, T. Fukaya, M. Krbal, T. Yagi, and 688 J. Tominaga, Nat. Nanotechnol. 6, 501 (2011).

$689{ }^{16}$ A. L. Lacaita and D. J. Wouters, Phys. Status Solidi A 205, 2281 (2008).

$690{ }^{17}$ H. P. Wong, S. Raoux, S. Kim, J. Liang, J. P. Reifenberg, B. Rajendran,

691 M. Asheghi, and K. E. Goodson, Proc. IEEE 98, 2201 (2010).

$692{ }^{18}$ S. Raoux, W. Wełnic, and D. Ielmini, Chem. Rev. 110, 240 (2010).

693 19. Reifenberg, E. Pop, A. Gibby, S. Wong, and K. Goodson, in Thermal and 694 Thermomechanical Proceedings 10th Intersociety Conference on Phenomena in 695 Electronics Systems, 2006. ITHERM 2006 (IEEE, 2006), pp. 106-113.

$696{ }^{20}$ A. Faraclas, G. Bakan, L. Adnane, F. Dirisaglik, N. E. Williams, A. Gokirmak, 697 and H. Silva, IEEE Trans. Electron Devices 61, 372 (2014).

$698{ }^{21}$ D. L. Kencke, I. V. Karpov, B. G. Johnson, S. J. Lee, D. Kau, S. J. Hudgens,

699 J. P. Reifenberg, S. D. Savransky, J. Zhang, M. D. Giles, and G. Spadini, in 2007

700 IEEE International Electron Devices Meeting (IEEE, 2007), pp. 323-326.

$701{ }^{22}$ J. P. Reifenberg, D. L. Kencke, and K. E. Goodson, IEEE Electron Device Lett. 702 29, $1112(2008)$.

$703{ }^{23}$ J. P. Reifenberg, K. Chang, M. A. Panzer, S. Kim, J. A. Rowlette, M. Asheghi, 704 H. P. Wong, and K. E. Goodson, IEEE Electron Device Lett. 31, 56 (2010).

$705{ }^{24}$ D. G. Cahill, H. E. Fischer, T. Klitsner, E. T. Swartz, and R. O. Pohl, J. Vac. 706 Sci. Technol. A 7, 1259 (1989).

$707{ }^{25}$ D. G. Cahill, Rev. Sci. Instrum. 61, 802 (1990)

$708{ }^{26}$ J. H. Kim, A. Feldman, and D. Novotny, J. Appl. Phys. 86, 3959 (1999).

$709{ }^{27} \mathrm{~L}$. Shi and A. Majumdar, ASME J. Heat Transfer 124, 329 (2002).

$710{ }^{28}$ B. Cretin, S. Gomes, N. Trannoy, and P. Vairac, Scanning Thermal Microscopy,

711 Microscale and Nanoscale Heat Transfer Topics (Springer-verlag, Berlin, 2007).

$712{ }^{29}$ H. M. Pollock and A. Hammiche, J. Phys. D: Appl. Phys. 34, R23 (2001).

$713{ }^{30}$ H. Fischer, Thermochim. Acta 425, 69 (2005).

$714{ }^{31} \mathrm{M}$. Nonnenmacher and H. K. Wickramasinghe, Appl. Phys. Lett. 61, 168

715 (1992).

$716{ }^{32}$ S. Lefèvre and S. Voltz, Rev. Sci. Instrum. 76, 033701 (2005).

$717{ }^{33}$ A. Majumdar, Annu. Rev. Mater. Sci. 29, 505 (1999).

$718{ }^{34}$ A. Majumdar, J. P. Carrejo, and J. Lai, Appl. Phys. Lett. 62, 2501 (1993).

$719{ }^{35}$ R. Aster, B. Borchers, and C. Thurber, Parameter Estimation and Inverse

720 Problems (Elsevier Science, 2018).

$721{ }^{36}$ S. Deemyad and I. F. Silvera, Rev. Sci. Instrum. 79, 086105 (2008).

$722{ }^{37}$ J.-L. Battaglia, A. Kusiak, V. Schick, A. Cappella, C. Wiemer, M. Longo, and 723 E. Varesi, J. Appl. Phys. 107, 044314 (2010).

$724{ }^{38}$ J.-L. Battaglia, V. Schick, C. Rossignol, A. Kusiak, I. Aubert, A. Lamperti, and

725 C. Wiemer, Appl. Phys. Lett. 102, 181907 (2013).

$726{ }^{39}$ J. J. Moré, in Numerical Analysis, edited by G. A. Watson (Springer, Berlin,

727 1978), pp. 105-116.

$728{ }^{40}$ R. H. Byrd, J. C. Gilbert, and J. Nocedal, Math. Program. 89, 149 (2000).

$729{ }^{41}$ W. K. Hastings, Biometrika 57, 97 (1970).

$730 \quad{ }^{42}$ R. D. Cowan, J. Appl. Phys. 32, 1363 (1961).

$731{ }^{43}$ P.-E. Nordal and S. O. Kanstad, Phys. Scr. 20, 659 (1979).

$732{ }^{44}$ J. Ishii, Y. Shimizu, K. Shinzato, and T. Baba, Int. J. Thermophys. 26, 1861 733 (2005)

$734{ }^{45}$ J.-L. Battaglia, A. Kusiak, M. Bamford, and J.-C. Batsale, Int. J. Therm. Sci. 45, 7351035 (2006).

$736{ }^{46}$ N. Horny, M. Chirtoc, A. Fleming, G. Hamaoui, and H. Ban, Appl. Phys. Lett. 737 109, 033103 (2016).

$738{ }^{47}$ H. G. Walther and T. Kitzing, J. Appl. Phys. 84, 1163 (1998).

$739{ }^{48}$ A. Mandelis, J. Batista, and D. Shaughnessy, Phys. Rev. B 67, 205208 (2003).

$740{ }^{49}$ S. Paoloni and D. Fournier, Rev. Sci. Instrum. 74, 523 (2003).

$741{ }^{50}$ S. André, B. Rémy, D. Maillet, A. Degiovanni, and J.-J. Serra, J. Appl. Phys. 742 96, 2566 (2004)

$743{ }^{51}$ M. Depriester, P. Hus, S. Delenclos, and A. H. Sahraoui, Rev. Sci. Instrum. 76, $744074902(2005)$.
${ }^{\mathbf{5 2}}$ R. Fuente, E. Apiñaniz, A. Mendioroz, and A. Salazar, J. Appl. Phys. 110, 745 033515 (2011). ${ }^{53}$ K. Ghosh, A. Kusiak, P. Noé, M.-C. Cyrille, and J.-L. Battaglia, Phys. Rev. B 747 101, 214305 (2020). 748 ${ }^{54}$ A. Kusiak, J.-L. Battaglia, P. Noé, V. Sousa, and F. Fillot, J. Phys.: Conf. Ser. 749 745, 032104 (2016). ${ }^{\mathbf{5 5}}$ J.-L. Battaglia, A. Kusiak, C. Gaborieau, Y. Anguy, H. T. Nguyen, C. Wiemer, 751 R. Fallica, D. Campi, M. Bernasconi, and M. Longo, Phys. Status Solidi RRL 10, 752 544 (2016).

56.-L. Battaglia, A. Kusiak, A. Saci, R. Fallica, A. Lamperti, and C. Wiemer, 754 Appl. Phys. Lett. 105, 121903 (2014). 755

${ }^{57}$ W. S. Capinski and H. J. Maris, Rev. Sci. Instrum. 67, 2720 (1996). 756

${ }^{58}$ M. G. Burzo, P. L. Komarov, and P. E. Raad, J. Heat Transfer 124, 1009757 (2002). ${ }^{{ }^{59}}$ T. Baba, K. Ishikawa, T. Yagi, and N. Taketoshi, arXiv e-prints, arXiv:0709. 759 1845 (2007). $\quad 760$ ${ }^{60}$ A. J. Schmidt, X. Chen, and G. Chen, Rev. Sci. Instrum. 79, 114902761 (2008). 762 ${ }^{61}$ S. Dilhaire, G. Pernot, G. Calbris, J. M. Rampnoux, and S. Grauby, J. Appl. 763 Phys. 110, 114314 (2011). 764 ${ }^{62}$ H.-K. Lyeo, D. G. Cahill, B.-S. Lee, J. R. Abelson, M.-H. Kwon, K.-B. Kim, 765 S. G. Bishop, and B.-K. Cheong, Appl. Phys. Lett. 89, 151904 (2006). 766 ${ }^{63}$ M. Kuwahara, O. Suzuki, Y. Yamakawa, N. Taketoshi, T. Yagi, P. Fons, 767 T. Fukaya, J. Tominaga, and T. Baba, Microelectron. Eng. 84, 1792 (2007). 768 ${ }^{64}$ J. P. Reifenberg, M. A. Panzer, S. Kim, A. M. Gibby, Y. Zhang, 769 S. Wong, H.-S. P. Wong, E. Pop, and K. E. Goodson, Appl. Phys. Lett. 91, 770 111904 (2007).

${ }^{65} \mathrm{Q}$. Li, J. Wei, H. Sun, K. Zhang, Z. Huang, and L. Zhang, Sci. Rep. 7, 13747772 (2017).

${ }^{66}$ R. J. Warzoha, B. F. Donovan, N. T. Vu, J. G. Champlain, S. Mack, and 774 L. B. Ruppalt, Appl. Phys. Lett. 115, 023104 (2019). 775 ${ }^{67}$ E. Bozorg-Grayeli, J. P. Reifenberg, K. W. Chang, M. Panzer, and 776 K. E. Goodson, in 2010 12th IEEE Intersociety Conference on Thermal and 777 Thermomechanical Phenomena in Electronic Systems (IEEE, 2010), pp. 1-7. 778 ${ }^{68}$ C. Kim, D.-S. Suh, K. H. P. Kim, Y.-S. Kang, T.-Y. Lee, Y. Khang, and 779 D. G. Cahill, Appl. Phys. Lett. 92, 013109 (2008). 780 ${ }^{69}$ W. J. Parker, R. J. Jenkins, C. P. Butler, and G. L. Abbott, J. Appl. Phys. 32, 781 1679 (1961)

${ }^{70}$ J. A. Cape and G. W. Lehman, J. Appl. Phys. 34, 1909 (1963).

${ }^{71}$ R. D. Cowan, J. Appl. Phys. 34, 926 (1963).

${ }^{72}$ J. T. Schriempf, Rev. Sci. Instrum. 43, 781 (1972).

${ }^{73}$ T. Azumi and Y. Takahashi, Rev. Sci. Instrum. 52, 1411 (1981).

${ }^{74}$ Y. Takahashi, T. Azumi, and M. Kanno, Netsu Sokutei 8, 62 (1981).

${ }^{75}$ A. Degiovanni, G. Sinicki, and M. Laurent, "Heat pulse thermal diffusivity 788 measurements-thermal properties temperature dependence and non-uniformity 789 of the pulse heating," in Thermal Conductivity 18, edited by T. Ashworth and 790 D. R. Smith (Springer US, Boston, MA, 1985), pp. 537-551.

${ }^{76}$ J. J. Hoefler and R. E. Taylor, Int. J. Thermophys. 11, 1099 (1990).

${ }^{77}$ T. Baba, M. Kobayashi, A. Ono, J. Hong, and M. Suliyanti, Thermochim. Acta 793 218, 329 (1993).

${ }^{78}$ T. Baba and A. Ono, Meas. Sci. Technol. 12, 2046 (2001). G. Labudova, and W. Hohenauer, Int. J. Thermophys. 23, 1157796 (2002). ${ }^{80}$ D. Maillet, S. André, J.-C. Batsale, A. Degiovanni, and C. Moyne, Thermal 798 Quadrupoles: Solving the Heat Equation Through Integral Transforms (Wiley, 799 2000).

${ }^{\mathbf{8 1}}$ J.-L. Battaglia, E. Ruffio, A. Kusiak, C. Pradere, E. Abisset, S. Chevalier, 801 A. Sommier, and J.-C. Batsale, Measurement 158, 107691 (2020). 